\title{
A Study on Charging Center Allocation Problem in EV Distributed Battery Swapping Network
}

\author{
Gumpanat Keardkeawfa, Yu Song, and Mingzhe Li
}

\begin{abstract}
In the near future, electric vehicles (EVs) will play an important role in the automobile industry, because they can reduce $\mathrm{CO}_{2}$ emissions and the use of fossil fuel. The largest challenge in popularizing EVs is the charging infrastructure. A potential solution is a distributed battery-swapping network, where depleted batteries are replaced at swapping stations. Then, the uncharged batteries are refilled at district-charging centers and sent back to battery-swapping stations. In this study, we study the problem of allocating charging centers in distributed battery-swapping networks and propose solving it using genetic algorithms. Through numerical examples, we illustrate the effectiveness of our proposed algorithm.
\end{abstract}

Index Terms-Electric vehicle, distributed battery-swapping network, charging center allocation, genetic algorithm.

\section{INTRODUCTION}

Electric vehicles (EVs) are a promising technology that drastically reduces the environmental burden of road transportation. Specifically, they can reduce $\mathrm{CO}_{2}$ emissions of passenger cars and light commercial vehicles, as well as emissions of pollutants and noise.

However, EVs are still far from proven technology. In particular, there exist many uncertainties with respect to crucial issues such as [1]:

1) Battery technology (energy capacity in relation to vehicle range and charging speed).

2) Durability, availability, and environment (impacts of materials).

3) Well-to-wheel impacts on emissions.

4) Interaction with electricity generation.

5) Cost of a business model in large-scale cases.

Among these issues, battery technology is considered as the most important one. A solution that was proposed to address it is a battery swap system, where drivers simply drive to a swapping station and swap a depleted battery with a full one. The battery-swapping operation only takes a few minutes. The advantages of a swapping system are (a) extending the driving range of EVs, (b) reducing the initial cost to buy an EV, and (c) upgrading batteries without additional cost. Several companies, such as the Better Place

Manuscript received November 5, 2012; revised January 20, 2013.

G. Keardkeawfa is with the Department of Management and Systems Engineering, Graduate School of Engineering, Fukuoka Institute of Technology, Fukuoka, Japan (e-mail: gumpanat.k@gmail.com).

Y. Song is with the Department of System Management, Faculty of Information Engineering, Fukuoka Institute of Technology, Fukuoka, Japan (e-mail: song @ fit.ac.jp).

M. Li is with the Department of Industrial Economics, Faculty of Economics, Fukuoka University, Fukuoka, Japan (e-mail: lmz@fukuoka-u.ac.jp).
Company, are implementing this business model in the United States, Japan, and other countries [2].

The distributed battery-swapping network (DBSN) is a larger-scale version of the battery-swapping system. The largest power company in China, State Grid Corporation, in conjunction with other power companies is promoting this network in China. In a DBSN, depleted EV batteries are replaced at battery-swapping stations, which are then sent to district-charging centers. When the depleted batteries are refilled, they are sent back to battery-swapping stations.

In [3], He et al. studied the problem of allocating charging centers in DBSNs. They formulated the problem as a mathematical model and proposed its solution by using particle swarm optimization (PSO). However, the solution obtained in their numerical example was not feasible. Hence, it is not clear if PSO effectively solves this model.

In this study, we use the same mathematical model as in [3] and propose a solution using a genetic algorithm (GA). Numerical results show that if we are careful when generating the initial solutions, the GA can solve the model effectively. In addition, we conduct comprehensive numerical tests to obtain insights on planning a network in practice.

The remainder of this paper is organized as follows: we introduce some related literature in Section II. We describe the formulation of the mathematical model of allocation problem in Section III. In Section IV, we briefly introduce the genetic algorithm and discuss how to code chromosome to solve our problem. Then, we evaluate the performance through numerical experiment (Section V). In Section VI, we present our conclusions and possible future studies.

\section{LITERATURE REVIEW}

In [1], the authors discuss important issues in logistic planning for EVs, such as battery management, battery supply chain, battery-charging services and recycling regulations. The authors of [4] provide some statistics about the fuel economy of EVs, which are useful when implementing numerical tests. Liu and Guo [5] discuss factors that affect the popularization of EVs and formulate a simulation model, which they use to forecast their popularity. Their results predict that the automobile market will be gradually overtaken by EVs. They conclude that during the electrification of transportation, EVs will have a large impact in energy saving. In [6], Zhang et al. compare gasoline cars with EVs and propose a planning model for the charging facilities of EVs.

The study presented in [3] discusses the problem of allocating charging centers in DBSNs and forms the basis of our study. According to the State Grid Corporation of China, 
which is a proactive promoter of DBSNs, the concept of DBSNs is "Replacing batteries is primary, charging is complementary, charging concentrated, distributing unified" [7]. In DBSNs, the allocation of charging centers directly affects the collection/distribution cost of batteries. The authors of [3], under the assumptions that the positions of the swapping stations and the maximum supply of electricity of charging centers are given, formulated a mathematical model for this problem. Then, they solved the model using a discrete PSO. In Fig. 1, we present a flowchart of the procedure used to obtain the optimal solution. However, the "optimal" solution obtained in their numerical example is infeasible.

In this study, we investigate the same problem formulated in [3]. However, instead of using PSO to determine the locations of the battery-charging centers in the DBSN, we apply the GA.

\section{MODEL FORMULATION}

In this section, we formulate the optimal allocation problem in a mathematical model. The model is the same as that used in [3].

\section{A. Number of Charging Centers}

Assume that the information about the battery swapping stations is provided. First, we need to determine the number of charging centers in the DBSN. Let $n$ be the number of swapping stations and $V_{i}(i=1,2,3, \ldots, n)$ be the demand for electric power for station $i$. The daily electric power a charging center can supply is denoted by $\Omega_{\max }$. Then, the number of charging centers, $m$, required is obtained by

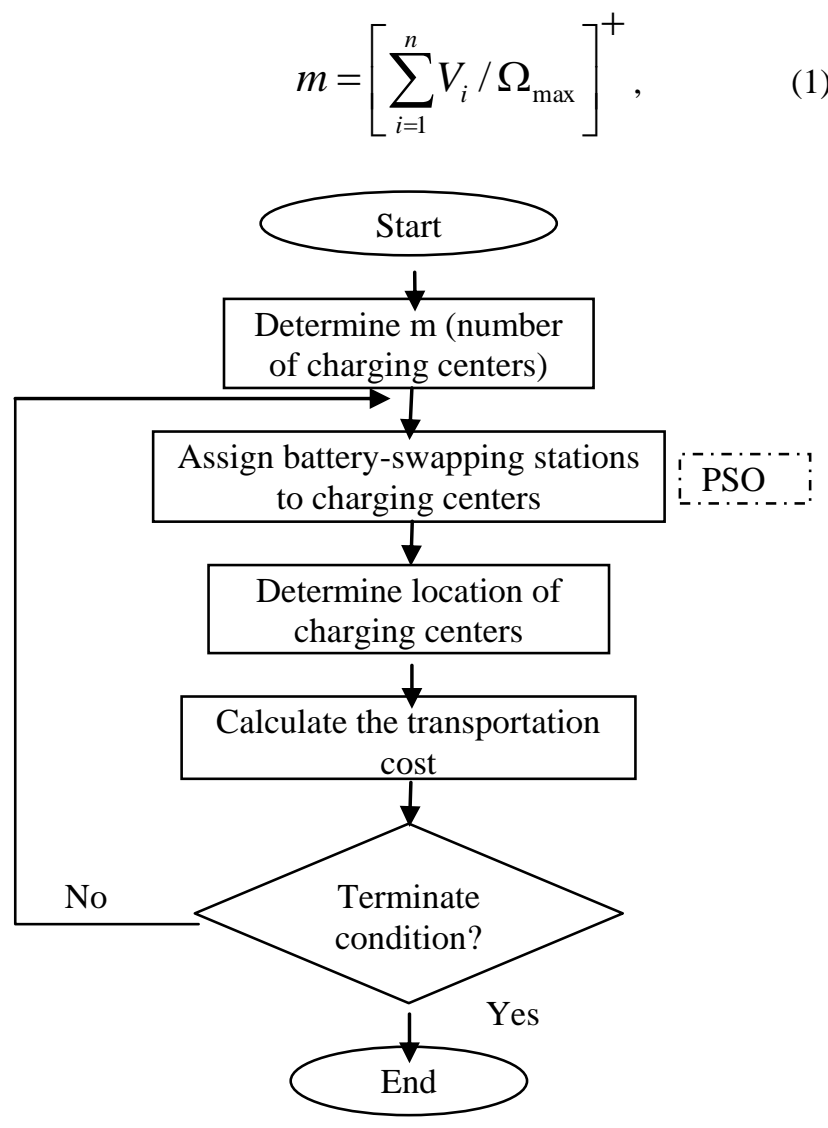

Fig. 1. Flowchart of procedure used to obtain the optimal solution. where the operator $[x]^{+}$designates the smallest integer greater than or equal to $x$. Next, we need to assign the $n$ swapping stations to the $m$ charging centers.

\section{B. Locations of Charging Centers}

Assume that we have assigned the battery-swapping stations to the charging centers. Then, we can use the center of gravity to determine the locations of the charging centers [3].

The coordinates of the locations of the battery-swapping stations are $\left(x_{1}, y_{1}\right),\left(x_{2}, y_{2}\right),\left(x_{3}, y_{3}\right), \ldots,\left(x_{n}, y_{n}\right)$. Let $W_{j}$ represent charging center $j$. We use $\delta_{j i} \in\{0,1\}$ ( $j \leq m, i \leq n$ ) to denote the number of assignments of battery-swapping stations to charging centers. Specifically, if station $i$ is assigned to charging center $j, \delta_{j i}=1$; otherwise $\delta_{j i}=0$.

By applying the center-of-gravity idea to the electric power and locations of the battery-swapping stations, we can obtain the locations of charging centers as follows:

$$
\begin{aligned}
& u_{j}=\left(\sum_{i=1}^{n} \delta_{j i} V_{i} x_{i}\right) /\left(\sum_{i=1}^{n} \delta_{j i} V_{i}\right), \\
& v_{j}=\left(\sum_{i=1}^{n} \delta_{j i} V_{i} y_{i}\right) /\left(\sum_{i=1}^{n} \delta_{j i} V_{i}\right),
\end{aligned}
$$

where $\left(u_{j}, v_{j}\right)$ are the coordinates of the charging center $j$.

\section{Objective Function}

Here we only consider the transportation cost between swapping stations and charging centers. The construction cost of the charging centers is neglected. Therefore, to obtain the optimal assignment between battery-swapping stations and charging centers, we use the following objective function:

$$
\text { Minimize } \left.C=\sum_{j=1}^{m} \sum_{i=1}^{n} \delta_{j i} V_{i} \sqrt{\left(u_{j}-x_{i}\right)^{2}+\left(v_{j}-y_{i}\right.}\right)^{2}
$$

\section{GENETIC AlgorithM}

In this section, we briefly introduce the basic idea of GAs and describe the genetic representation used in this study (subsection B). Moreover, we discuss how to construct the initial solutions to obtain the optimal solution more effectively (subsection C).

\section{A. Introduction to GAs}

The GA is an "intelligent" heuristic search algorithm that can be applied to various combinatorial optimization problems. The GA emulates Darwin's principle of natural selection, i.e., during the course of evolution, natural populations evolve according to the principle of natural selection and 'survival of the fittest'. Individuals that are more successful in adapting to their environment have a 
better chance of surviving and reproducing, whereas individuals that are less fit are eliminated. The GA process is described in Fig. 2.

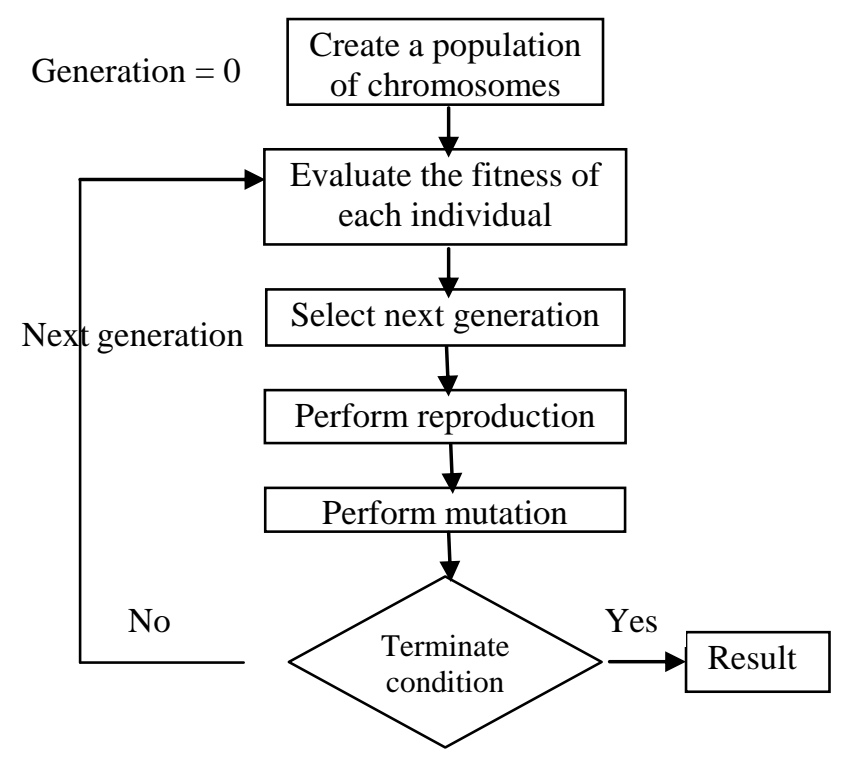

Fig. 2. Genetic algorithm process.

\section{B. Genetic Representation}

When solving a problem using a GA, it is important to design the appropriate genetic representation of solutions. In this study, we use the following coding scheme to represent solutions.

Assume that battery stations $1,2,3,4,5,6, \ldots, n$ are assigned to $W_{l}, W_{1}, W_{2}, W_{1}, W_{3}, W_{3}, \ldots, W_{2}$, respectively. Hence, $W_{1}$ (charging center 1 ) supplies batteries to stations 1,2 , and $4, W_{2}$ supplies batteries to stations 3 and $n$, and $W_{3}$ supplies batteries to stations 5 and 6 . Therefore, the chromosome that represents this solution is (112133...2) .

The advantage of this representation is that it ensures ther assignment of every battery-swapping station to exactly one charging center. The disadvantage is that it allows the generation of infeasible solutions. In particular, if the constraints are stringent, this representation will generate a large number of infeasible solutions.

\section{Improved Initialization Procedure for $G A$}

In the next section, we show that the constraints applied to the assignment of battery-swapping stations are not flexible. Therefore, if we randomly generate initial solutions, a large number of them will be infeasible, and consequently, we may not be able to obtain the optimal solution. To avoid this problem, we propose generating initial solutions as follows:

Step 1: Randomly select $j(j=1,2, \ldots, m)$ and sort the charging centers in the order $j, j+1, \ldots, m, 1,2, \ldots, j-1$.

Step 2: Randomly select $i(i=1,2, \ldots, n)$ and sort the swapping stations in the order $i, i+1, \ldots, n, 1,2, \ldots, i-1$.

Step 3: Assign swapping station $i$ to $W_{j}$.

Step 4-1: Calculate the demand for electric power for $W_{j}$.

Step 4-2: If the electric power required by $W_{j}$ does not exceed the electric power limit, assign station $i$ to $W j$. Otherwise, re-assign station $i$ to the next unassigned center.

Step 4-3: Repeat Steps 2 to 4-2 for all swapping stations.
Step 5: Select the next charging center and go to Step 3. Repeat Step 5 for all centers.

Step 6: If more initial solutions are required, go to Step 1.

Using this algorithm, we generate only feasible initial solutions. As a result, the GA converges faster and obtains better solutions.

\section{NUMERICAL RESULTS}

In this section, we conduct numerical simulations to test our proposed algorithm. First, we compare our algorithm against the numerical example presented in [3]. Then, we conduct a comprehensive test to investigate the sensitivity of our algorithm and obtain insights on planning a network.

\section{A. Case 1}

In this test, we consider the example used in [3]. Assume that a certain district contains 22 battery-swapping stations. The locations and electric power requirements of each battery-swapping station are listed in Table I. Moreover, the maximal electric power supplied from each charging center is set to $\Omega_{\max }=7000 \mathrm{kWh}$.

We apply the GA optimization technique to solve this model. The GA parameters used are as follows: population size $=100$, number of generations $=10000$, number of crossover points $=2$, mutation rate $=0.05$.

Table II shows the results obtained for the locations and assigned stations of each charging center. The number of charging centers is $4(m=4)$, and the optimal value of the objective function is $C=445.05$. In Fig. 3, we plot the locations of the charging centers listed in Table II.

TABLE I: LOCATIONS AND DEMANDS FOR ELECTRIC POWER OF EACH BATTERY-SWAPPING STATION (BSS) FOR CASE 1

\begin{tabular}{|c|c|c|c|}
\hline BSS ID & \multicolumn{2}{|c|}{ Location } & Electric Power (kWh) \\
\hline 1 & 34.50 & 95.47 & 1260 \\
\hline 2 & 41.03 & 87.32 & 960 \\
\hline 3 & 22.34 & 81.20 & 1200 \\
\hline 4 & 51.67 & 78.32 & 960 \\
\hline 5 & 44.37 & 76.37 & 1080 \\
\hline 6 & 27.87 & 65.59 & 960 \\
\hline 7 & 63.24 & 72.37 & 900 \\
\hline 8 & 18.72 & 62.94 & 1200 \\
\hline 9 & 68.12 & 58.32 & 960 \\
\hline 10 & 21.32 & 33.83 & 1230 \\
\hline 11 & 77.35 & 42.86 & 960 \\
\hline 12 & 33.13 & 33.19 & 960 \\
\hline 13 & 35.17 & 31.10 & 1200 \\
\hline 14 & 53.17 & 33.17 & 1020 \\
\hline 15 & 52.17 & 42.19 & 1200 \\
\hline 16 & 40.32 & 75.73 & 1440 \\
\hline 17 & 55.39 & 32.17 & 1440 \\
\hline 18 & 14.30 & 35.47 & 1500 \\
\hline 19 & 28.32 & 61.70 & 1920 \\
\hline 20 & 44.12 & 17.36 & 1440 \\
\hline 21 & 18.45 & 9.30 & 1470 \\
\hline 22 & 83.24 & 52.35 & 1920 \\
\hline
\end{tabular}


TABLE II: GA RESULTS FOR LOCATIONS OF CHARGING CENTERS AND ASSIGNMENTS OF BATTERY-SWAPPING STATIONS FOR CASE 1

\begin{tabular}{|l|l|l|l|}
\hline $\begin{array}{c}\text { Charging } \\
\text { Center ID }\end{array}$ & \multicolumn{2}{|c|}{ Location } & \multicolumn{1}{|c|}{$\begin{array}{c}\text { Assigned battery-swapping } \\
\text { stations }\end{array}$} \\
\hline 1 & 36.11 & 31.33 & $9,10,12,13,14,21$ \\
\hline 2 & 25.07 & 61.90 & $3,5,8,18,19$ \\
\hline 3 & 42.18 & 79.43 & $1,2,4,6,7,16$ \\
\hline 4 & 63.21 & 37.87 & $11,15,17,20,22$ \\
\hline
\end{tabular}

In Fig. 4, we compare the convergence of GA with and without the improved initialization procedure presented in subsection V-C. In this example, we see that if we do not use the improved initialization procedure, GA does not obtain the optimal solution. Hence our initialization algorithm is quite effective.

\section{B. Case 2}

In this case, we test the performance of our proposed algorithm by varying the number of battery-swapping stations.

First, we perform a numerical tested with 40 stations. Similar to Case 1, the locations and electric power requirements of each battery-swapping station are given. Similarly, the maximal electric power supplied by each charging center is set to $\Omega_{\max }=7000 \mathrm{kWh}$. For this test, we use the following GA parameters: population size $=1000$, number of iterations $=20000$, number of crossover points $=2$, mutation rate $=0.05$.

Table III shows the result obtained for the locations and assigned stations of each charging center. In this case, the number of charging centers is $8(m=8)$, and the optimal value of the objective function is $C=598.96$.

In the next test performed, we varied the number of battery-swapping stations from 15 to 80 . The GA parameters used are as follows: population size $=100$, number of iterations $=10000$, number of crossover points $=2$, mutation rate $=0.05$. From Fig. 5, we see that the run time of GA increases approximately linearly to the number of stations.

\section{Case 3}

In this test, we add an additional charging center to the problem. Hence, we effectively relax the constraints of the model. Next, we compare the transportation cost with that of the original case (Case 1).

In this test, we use the same number of battery-swapping stations, locations and battery requirements of each swapping station and the maximal electric power of each charging center as in Case 1. The only difference is, instead of using (1) to compute the number of charging centers, we use

$$
m=\left[\sum_{i=1}^{n} V_{i} / \Omega_{\max }\right]^{+}+1
$$

Table IV shows the GA result for the locations and assigned stations of each charging center. In this case, the number of charging centers is $5(m=5)$, and the optimal value of the objective function is $C=274.96$ (i.e., $61 \%$ of the value obtained in Case 1). This implies that the addition of one charging center reduces the transportation cost by $1 / 3$.

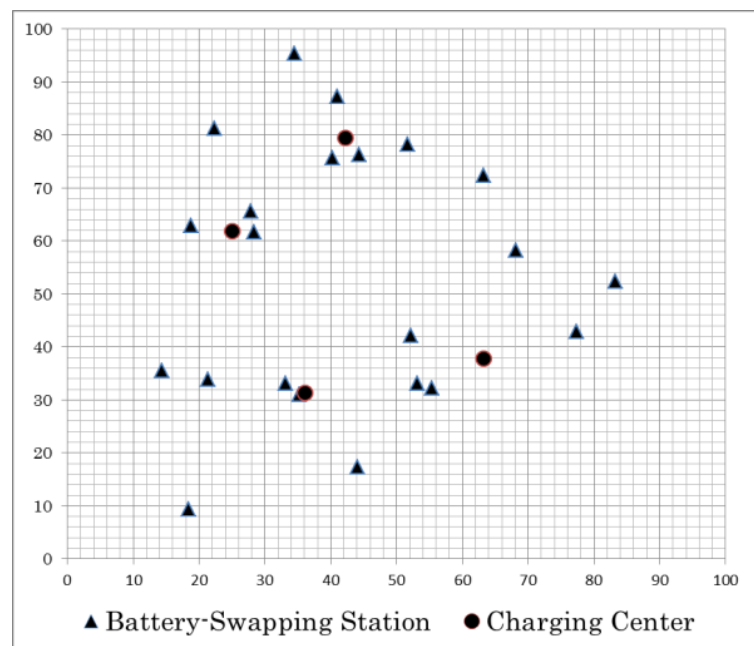

Fig. 3. Locations of charging centers and swapping stations for Case 1 .

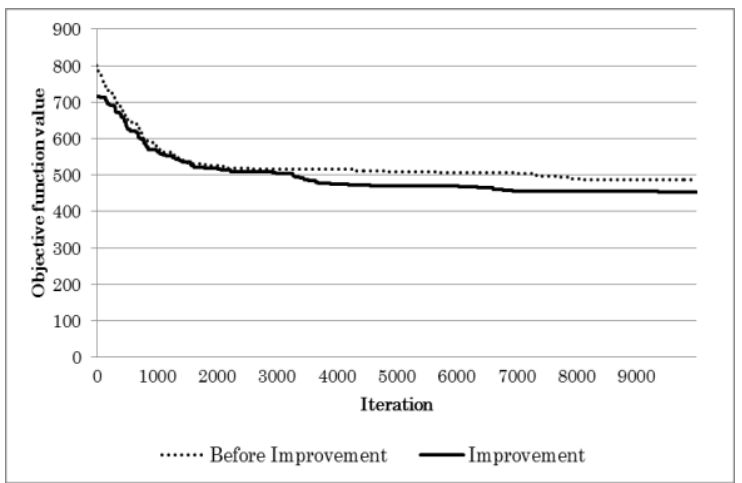

Fig. 4. Convergence performance of GA for Case 1.

TABLE III: GA RESULTS FOR LOCATIONS OF CHARGING CENTERS AND BATTERY-SWAPPING STATION ASSIGNMENTS FOR CASE 2

\begin{tabular}{|l|l|l|l|}
\hline $\begin{array}{c}\text { Charging } \\
\text { Center } \\
\text { ID }\end{array}$ & \multicolumn{2}{|c|}{ Location } & \multicolumn{1}{|c|}{$\begin{array}{c}\text { Assigned battery-swapping } \\
\text { stations }\end{array}$} \\
\hline 1 & 92.55 & 67.17 & $27,28,30,38$, \\
\hline 2 & 56.99 & 44.04 & $7,9,14,15,17,25$, \\
\hline 3 & 65.26 & 88.47 & $4,23,32,35,37$, \\
\hline 4 & 36.60 & 82.47 & $1,2,3,5,16$, \\
\hline 5 & 33.59 & 14.37 & $20,21,31,34,36$, \\
\hline 6 & 36.94 & 34.59 & $10,12,13,26,29$, \\
\hline 7 & 21.35 & 60.58 & $6,8,18,19,39$, \\
\hline 8 & 78.45 & 51.12 & $11,22,24,33,40$, \\
\hline
\end{tabular}

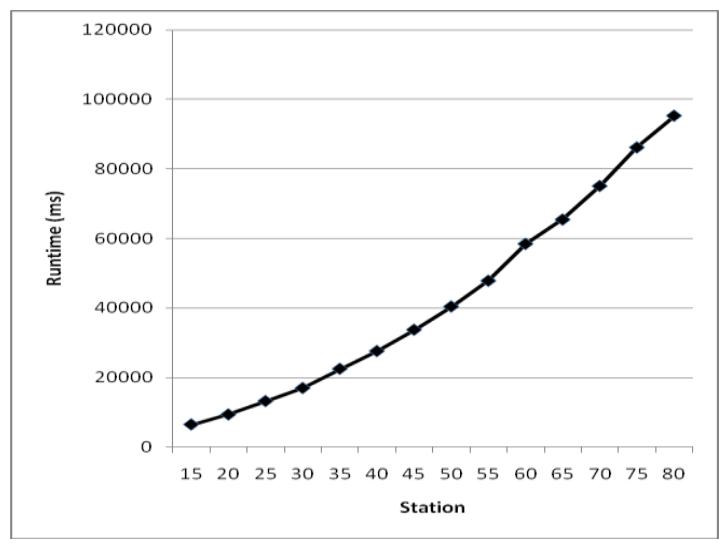

Fig. 5. Run time of GA as a function of the number of stations.

By comparing the transportation cost of Case 3 with that in Case 1, we can extract additional information that could be 
useful for location planning in DBSNs. In particular, in addition to transportation costs, our objective function could account for the construction costs of charging centers and be used to determine the optimal number of charging centers for a DBSN.

TABLE IV: GA RESULTS FOR LOCATIONS OF CHARGING CENTERS AND ASSIGNMENTS OF BATTERY-SWAPPING STATIONS FOR CASE 3.

\begin{tabular}{|l|l|l|l|}
\hline $\begin{array}{c}\text { Charging } \\
\text { Center } \\
\text { ID }\end{array}$ & \multicolumn{2}{|c|}{ Location } & \multicolumn{1}{|c|}{$\begin{array}{c}\text { Assigned battery-swapping } \\
\text { stations }\end{array}$} \\
\hline 1 & 24.70 & 67.12 & $3,6,8,19$ \\
\hline 2 & 75.19 & 55.44 & $7,9,11,22$ \\
\hline 3 & 41.71 & 82.07 & $1,2,4,5,16$ \\
\hline 4 & 23.40 & 27.94 & $10,12,13,18,21$ \\
\hline 5 & 51.01 & 30.55 & $14,15,17,20$ \\
\hline
\end{tabular}

\section{CONCLUSIONS}

In this study, we studied the optimal allocation problem of charging centers for EVs in a DBSN and solved it using a GA. Numerical results showed that if we carefully select the initial solutions, our proposed algorithm is effective. Furthermore, we performed several numerical simulations to obtain insights on planning a network.

When we use only feasible initial solutions, our GA tends to generate more feasible solutions. However, the crossover and mutation operations in each generation produce several infeasible solutions. Hence, we could improve our algorithm by reducing the number of infeasible solutions generated in each generation.

As a future study, we could also improve our algorithm by considering other types of costs in our objective function, such as construction cost. This enhancement would make our approach more useful in practical situations.

\section{REFERENCES}

[1] H. M. Wang, H. F. Xu, and A. K. Jones, "Crucial issues in logistic planning for electric vehicle battery application service," in Proc. 2010 International Conf. Optoelectronics and Image Processing, pp. 362-366, Nov. 2010

[2] Better Place. [Online]. Available: www.betterplace.com, January 2013

[3] Z. Y. He, Y. H. Zhou, N. Liang, K. W. Yue, C. Cheng, and Y. Yang, "The location studies of charging station for replacing batteries," in Proc 2011 International Conf. Advanced Power System Automation and Protection, pp. 2013-2017, Oct. 2011.

[4] MIT Electric Vehicle Team. (2008). Fuel Economy Numbers for Electric Vehicles. [Online]. Available: http://web.mit.edu/evt/ summary_mpgge.pdf .

[5] H. M. Liu and T. G. Guo, "Model to forecast the popularity of electric vehicles," in Proc 2nd International Conf. Mechanic Automation and Control Engineering, pp .417-421, July 2011.

[6] J. C. Zhang, Y. X. Liu, Y. L. Niu, N. X. Song, and X. L. Li, "Study on the planning model of electric vehicle charging facilities," Power and Energy Engineering Conf., 2012 Asia-Pacific, Mar. 2012, pp. 1-4.

[7] DBSN is a promising model for EV development. (January 2013). [Online]. Available: http://www.hexingfd.com/sj/tn-1/cx-1/30.html.

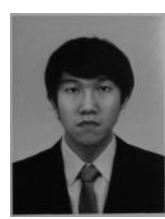

Gumpanat Keardkeawfa was born in Bangkok, Thailand. He earned his Bachelor degree from Chulalongkorn University, Thailand. Currently, he is a Master's Degree student at Fukuoka Institute of Technology, Japan.

Yu Song was born in China. He earned his Ph.D. at Tohoku University, Japan. Currently, he is a professor of Fukuoka Institute of Technology, Japan.

Mingzhe Li was born in China. He earned his Ph.D. at the University of Tokyo, Japan. Currently, he is a professor of Fukuoka University, Japan. 